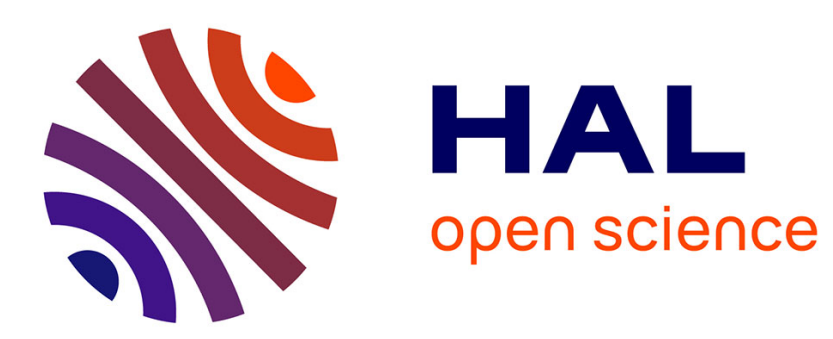

\title{
New Asymptotic Behavior of the Surface-Atom Force out of Thermal Equilibrium
}

\author{
Mauro Antezza, Lev P Pitaevskii, Sandro Stringari
}

\section{To cite this version:}

Mauro Antezza, Lev P Pitaevskii, Sandro Stringari. New Asymptotic Behavior of the Surface-Atom Force out of Thermal Equilibrium. Physical Review Letters, 2005, 95 (11), pp.113202. 10.1103/PhysRevLett.95.113202 . hal-02962766

\section{HAL Id: hal-02962766 https://hal.science/hal-02962766}

Submitted on 9 Oct 2020

HAL is a multi-disciplinary open access archive for the deposit and dissemination of scientific research documents, whether they are published or not. The documents may come from teaching and research institutions in France or abroad, or from public or private research centers.
L'archive ouverte pluridisciplinaire HAL, est destinée au dépôt et à la diffusion de documents scientifiques de niveau recherche, publiés ou non, émanant des établissements d'enseignement et de recherche français ou étrangers, des laboratoires publics ou privés. 


\title{
New Asymptotic Behavior of the Surface-Atom Force out of Thermal Equilibrium
}

\author{
Mauro Antezza, ${ }^{1}$ Lev P. Pitaevskii, ${ }^{1,2}$ and Sandro Stringari ${ }^{1,3}$ \\ ${ }^{1}$ Dipartimento di Fisica, Università di Trento and BEC Center, INFM-CNR, Via Sommarive 14, I-38050 Povo, Trento, Italy \\ ${ }^{2}$ Kapitza Institute for Physical Problems, ulitza Kosygina 2, 119334 Moskow, Russia \\ ${ }^{3}$ École Normale Supérieure and Collège de France, Laboratoire Kastler Brossel, 24 Rue Lhomond 75231 Paris, France
}

(Received 29 April 2005; published 7 September 2005)

\begin{abstract}
The Casimir-Polder-Lifshitz force felt by an atom near the surface of a substrate is calculated out of thermal equilibrium in terms of the dielectric function of the material and of the atomic polarizability. The new force decays like $1 / z^{3}$ at large distances (i.e., slower than at equilibrium), exhibits a sizable temperature dependence, and is attractive or repulsive depending on whether the temperature of the substrate is higher or smaller than the one of the environment. Our predictions can be relevant for experiments with ultracold atomic gases. Both dielectric and metal substrates are considered.
\end{abstract}

DOI: 10.1103/PhysRevLett.95.113202

An intense effort has been devoted in recent years to study the force felt by an atom near the surface of a substrate (see, for example, [1,2] and references therein). These studies are motivated both by the possibility of technological applications [3] and by the fundamental search for stronger constraints on hypothetical nonNewtonian forces [4]. Experimental and theoretical research has also recently focused on the forces acting on ultracold atomic gases, including atomic beams [5-7], Bose-Einstein condensates [2,8-10], and degenerate Fermi gases [11].

The force generated by the surface contains, in general, two independent components. The first one is related to zero-point fluctuations of the electromagnetic field. At short distances $z$ (typically less than fractions of microns) this force behaves like $1 / z^{4}$ and is the analog of the van der Waals-London interatomic force. At larger distances the inclusion of relativistic retardation effects gives rise to a different $1 / z^{5}$ dependence characterizing the socalled Casimir-Polder regime [12-14]. Notice that both the van der Waals-London and Casimir-Polder forces depend on the temperature only through the dielectric properties of the substrate. The resulting temperature dependence is usually negligible.

The second component of the force is due to the thermal fluctuations of the electromagnetic field. This effect was first considered by Lifshitz [15] who employed the theory of electromagnetic fluctuations developed by Rytov [16]. We refer to it as to the Lifshitz force. At distances larger than the thermal photon wavelength $\lambda_{T}=\hbar c / k_{B} T$ (corresponding to $\sim 7.6 \mu \mathrm{m}$ at room temperature) this force decays, at thermal equilibrium, like $1 / z^{4}$, is attractive, is proportional to the temperature, and provides the leading contribution to the total force. Conversely, the zero-point fluctuation component prevails at smaller distances. In this Letter we are interested in the thermal component of the force. The Lifshitz force was originally evaluated at full thermodynamic equilibrium. A nontrivial issue is the study of the force out of thermal equilibrium $[17,18]$, characterizing configurations where the temperature of the substrate
PACS numbers: 34.50.Dy, 12.20. $-\mathrm{m}, 42.50 . \mathrm{Nn}, 42.50 . \mathrm{Vk}$

and the one of the surrounding walls located at large distances (hereafter called environment temperature) do not coincide. The problem is relevant both for practical reasons, due to the possibility of tuning the two temperatures independently, and for a better understanding of the interplay between zero-point and thermal fluctuation effects. A first important investigation of the surface-atom force out of thermal equilibrium was carried out by Henkel et al. [17] who calculated the force generated by a dielectric substrate at finite temperature by assuming that the environment temperature is zero. The principal motivation of that paper was the study of the force at short distances. In this Letter we show that, out of thermal equilibrium, the force acting on the atom exhibits a new asymptotic behavior, characterized by a $1 / z^{3}$ decay at large distances.

Let us consider an atom placed in vacuum at distance $z$ from the flat surface of a substrate made of a material with dielectric function $\varepsilon(\omega)=\varepsilon^{\prime}(\omega)+i \varepsilon^{\prime \prime}(\omega)$. We choose a coordinate system with the $x y$ plane coinciding with the interface and the $z$ axis such that the substrate occupies the region with $z<0$ and the vacuum the region with $z>0$. In this Letter we assume that the substrate is locally at thermal equilibrium at a temperature $T_{S}$, which can differ from the environment temperature $T_{E}$, the global system being out of thermal equilibrium, but in a stationary regime. The total electromagnetic field will be, in general, the sum of the radiation produced by the substrate and the one of the environment. In particular, this latter radiation will be partially absorbed and reflected from the substrate. The forces produced by the $T_{E}$ and $T_{S}$ components of the radiation add incoherently. In typical experiments with ultracold atomic gases the environment temperature is determined by the chamber containing the substrate and the trapped atoms. We further treat the atoms as being at zero temperature in the sense that the surrounding radiation is not able to populate their excited states, which are assumed to be located at energies $\hbar \omega_{\text {at }}$ much higher than the thermal energy:

$$
k_{B} T_{S}, \quad k_{B} T_{E} \ll \hbar \omega_{\mathrm{at}} .
$$


This condition is very well satisfied at ordinary temperatures. Actually the first optical resonance of $\mathrm{Rb}$ atoms corresponds to $1.8 \times 10^{4} \mathrm{~K}$. In general, the force acting on a neutral atom can be written as $[17,19]$

$$
\mathbf{F}(\mathbf{r}) \approx\left\langle d_{i}^{\text {ind }} \nabla E_{i}^{\mathrm{f}}(\mathbf{r})\right\rangle+\left\langle d_{i}^{\mathrm{f}} \nabla E_{i}^{\text {ind }}(\mathbf{r})\right\rangle,
$$

holding in lowest order perturbation theory with $i=x, y, z$. Here and in the following we use Einstein's summation convention. The first term describes the field fluctuations correlated with the induced atomic dipole moment while the second one involves the dipole fluctuations correlated with the induced electric field. At thermal equilibrium, where $T_{S}=T_{E} \equiv T$, the force can be conveniently written in the form

$$
F^{\mathrm{eq}}(T, z)=F_{0}(z)+F_{\mathrm{th}}^{\mathrm{eq}}(T, z),
$$

where we have separated the contribution $F_{0}(z)$ arising from the $T=0$ zero-point fluctuations and the one arising from the thermal fluctuations. At large distances the $T=0$ force exhibits the Casimir-Polder asymptotic behavior

$$
F_{0}(z)_{z \rightarrow \infty}=-\frac{3}{2} \frac{\hbar c \alpha_{0}}{\pi z^{5}} \frac{\varepsilon_{0}-1}{\varepsilon_{0}+1} \phi\left(\varepsilon_{0}\right)
$$

with the function $\phi\left(\varepsilon_{0}\right)$ defined, for example, in [2]. Conversely, the thermal force approaches the Lifshitz law

$$
F_{\mathrm{th}}^{\mathrm{eq}}(T, z)_{z \rightarrow \infty}=-\frac{3}{4} \frac{k_{B} T \alpha_{0}}{z^{4}} \frac{\varepsilon_{0}-1}{\varepsilon_{0}+1},
$$

which then provides the leading contribution to the total force. The asymptotic law (5) is reached at distances larger than the thermal wavelength $\lambda_{T}$. In the above equations $\alpha_{0}$ $\left(=47.3 \times 10^{-24} \mathrm{~cm}^{3}\right.$ for $\mathrm{Rb}$ atoms $)$ and $\varepsilon_{0}$ are, respectively, the static polarizability of the atom and the static dielectric function of the substrate. It is worth noticing that only the static optical properties enter the asymptotic laws (4) and (5), the corresponding dynamic effects becoming important only at shorter distances. It is also worth noticing that the asymptotic behavior of the thermal force has a classical nature, being independent of the Planck constant. The explicit behavior of the force (3) at all distances has been recently investigated in [2].

Let us now discuss the behavior of the force when the system is not in equilibrium (neq). Also in this case the force can be written as the sum of the zero temperature contribution $F_{0}(z)$ and of a thermal contribution which, however, will differ from $F_{\text {th }}^{\mathrm{eq}}$ if $T_{S} \neq T_{E}$ :

$$
F^{\text {neq }}\left(T_{S}, T_{E}, z\right)=F_{0}(z)+F_{\text {th }}^{\text {neq }}\left(T_{S}, T_{E}, z\right) .
$$

The purpose of this Letter is to calculate the force (6) and, in particular, to exploit its behavior at large distances. Let us first consider the case of a substrate at finite temperature $\left(T_{S}=T \neq 0\right)$ in the absence of the environment radiation $\left(T_{E}=0\right)$. This problem was solved by Henkel et al. [17] who obtained the result

$$
\begin{aligned}
F_{\mathrm{th}, \mathrm{nff}}^{\mathrm{neq}}(T, 0, z)= & \frac{\hbar}{2 \pi^{2}} \int_{0}^{\infty} d \omega \frac{\varepsilon^{\prime \prime}(\omega)}{e^{\hbar \omega / k_{B} T}-1} \operatorname{Re}[\alpha(\omega) \\
& \left.\times \int_{V_{S}} G_{i k}\left[\omega ; \mathbf{r}, \mathbf{r}_{1}\right] \partial_{z} G_{i k}^{*}\left[\omega ; \mathbf{r}, \mathbf{r}_{1}\right] d^{3} \mathbf{r}_{1}\right]
\end{aligned}
$$

for the thermal contribution to the force originating from the fluctuations of the field (ff). In Eq. (7) $G_{i k}$ is the Green function relative to the electromagnetic field. The variable $\mathbf{r}_{1}$ should be integrated on the volume $V_{S}$ occupied by the substrate, which provides the source of the thermal radiation. The argument $\mathbf{r}$ instead defines the position of the atom outside the substrate. The Green function $G_{i k}$ then reduces to its transmitted component [20].

The force (7) contains a repulsive coordinate independent wind part (arising from the transmitted propagating modes) proportional to $\alpha^{\prime \prime}(\omega)$ and produced by the absorption of photons by the atom, and a dispersive part (arising from the transmitted evanescent modes) proportional to $\alpha^{\prime}(\omega)$. Because of the condition (1) the wind contribution can be ignored and the real part $\alpha^{\prime}(\omega)$ can be replaced with its static $(\omega=0)$ value $\alpha_{0}$. Furthermore, also the terms arising from the dipole fluctuations can be ignored in the evaluation of the thermal force, which can be conveniently rewritten in the form $F_{\mathrm{th}}^{\mathrm{neq}}\left(T_{S}, 0, z\right)=4 \pi \alpha_{0} \partial_{z} U_{E}\left(T_{E}, 0, z\right)$ where $U_{E}=\left\langle E^{2}\right\rangle / 8 \pi$ is the thermal component of the electric energy density in vacuum. The dispersive component of the force (7) can be explicitly worked out by introducing the Fourier transform $g_{i k}\left[\omega ; \mathbf{K}, z_{a}, z_{b}\right]$ of the Green function $G_{i k}\left[\omega ; \mathbf{r}_{a}, \mathbf{r}_{b}\right]$ where $\mathbf{K}$ is the component of the electromagnetic wave vector parallel to the interface. By explicitly expressing the function $g_{i k}$ in terms of the transmitted Fresnel coefficients [20] and using the procedure described in [21] we find, after some lengthy algebra, the important result

$$
\begin{aligned}
F_{\mathrm{th}}^{\mathrm{neq}}(T, 0, z)= & -\frac{2 \sqrt{2} \hbar \alpha_{0}}{\pi c^{4}} \int_{0}^{\infty} d \omega \frac{\omega^{4}}{e^{\hbar \omega / k_{B} T}-1} \\
& \times \int_{1}^{\infty} d q q e^{-2 z \sqrt{q^{2}-1} \omega / c} \sqrt{q^{2}-1} \sqrt{\left|\varepsilon(\omega)-q^{2}\right|+\left(\varepsilon^{\prime}(\omega)-q^{2}\right)}\left(\frac{1}{\left|\sqrt{\varepsilon(\omega)-q^{2}}+\sqrt{1-q^{2}}\right|^{2}}\right. \\
& \left.+\frac{\left(2 q^{2}-1\right)\left(q^{2}+\left|\varepsilon(\omega)-q^{2}\right|\right)}{\left|\sqrt{\varepsilon(\omega)-q^{2}}+\varepsilon(\omega) \sqrt{1-q^{2}}\right|^{2}}\right),
\end{aligned}
$$

where we have introduced the dimensionless variable $q=K c / \omega$. Equation (8) provides the thermal force generated by the 
substrate in the absence of the environment radiation. In order to discuss the more general case $T_{S} \neq T_{E} \neq 0$, we make use of the additivity property of the thermal force which can be written, in general, as the sum of two contributions: $\quad F_{\mathrm{th}}^{\text {neq }}\left(T_{S}, T_{E}, z\right)=F_{\mathrm{th}}^{\text {neq }}\left(T_{S}, 0, z\right)+F_{\mathrm{th}}^{\text {neq }}\left(0, T_{E}, z\right)$, produced, respectively, by the radiation of the substrate and of the environment. Their additivity can be checked by evaluating separately the two contributions and verifying that their sum, at thermal equilibrium, reproduces the Lifshitz force $F_{\mathrm{th}}^{\mathrm{eq}}$ [22]. The full surface-atom force out of equilibrium can finally be written in the convenient form

$$
F^{\mathrm{neq}}\left(T_{S}, T_{E}, z\right)=F^{\mathrm{eq}}\left(T_{E}, z\right)+F_{\mathrm{th}}^{\mathrm{neq}}\left(T_{S}, 0, z\right)-F_{\mathrm{th}}^{\mathrm{neq}}\left(T_{E}, 0, z\right),
$$

where the equilibrium force $F^{\mathrm{eq}}(T, z)$ is given by (3) while $F_{\text {th }}^{\text {neq }}(T, 0, z)$ is defined by Eq. (8).

In Figs. 1-3, we show the explicit results for the potential energy, the force, and the gradient of the force obtained starting from Eq. (9) as a function of the distance from the surface for different choices of $T_{S}$ and $T_{E}$. Calculations have been done for a sapphire substrate and for rubidium atoms. For $F^{\mathrm{eq}}(T, z)$ we have used the predictions of [2]. The figure clearly shows that the thermal effects out of equilibrium are sizable (solid lines), thereby providing promising perspectives for future measurements of the surface-atom force at large distances. In particular, in order to increase the attractive nature of the force, it is much more convenient to heat the substrate by keeping the environment at room temperature (lower solid line) rather than heating the whole system (dashed line). When $T_{S}<$ $T_{E}$ (upper solid line), the force exhibits a characteristic change of sign reflecting a repulsive nature at large distances (see also discussion below). At short distances the thermal correction to the force becomes smaller and smaller and is determined by the temperature of the substrate. We have reported the results for the potential, for the

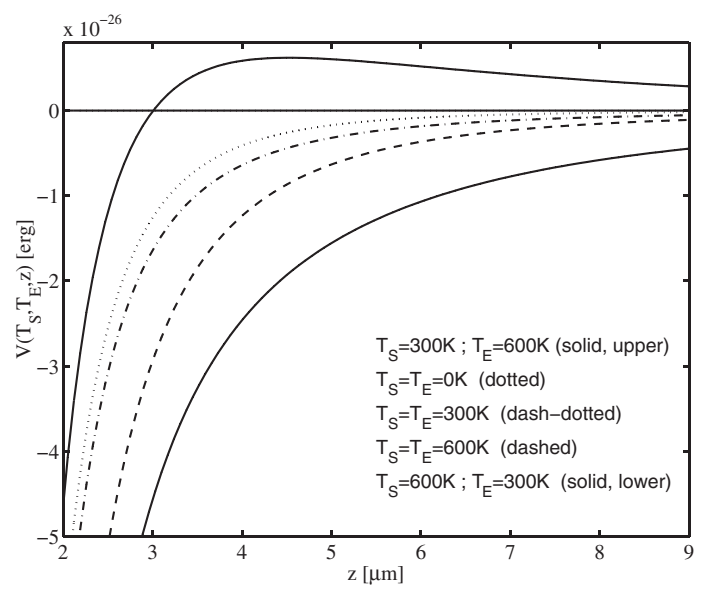

FIG. 1. Surface-atom potential energy $V^{\text {neq }}(z)=$ $-\int_{z}^{+\infty} d z^{\prime} F^{\text {neq }}\left(z^{\prime}\right)$ calculated from Eq. (9), for different thermal configurations. force, and for the gradient of the force because the corresponding predictions can be of interest for different types of experiments with ultracold gases. Experiments based on the study of the center of mass oscillation of a trapped gas are sensitive to the gradient of the force [2]. The corresponding frequency shifts produced by the surface-atom interaction have been recently measured [10] in conditions of thermal equilibrium in agreement with the predictions of theory [2]. Conversely, experiments based on Bloch oscillations are sensitive to the force itself $[11,23]$. Finally, one can also think at interference experiments with BoseEinstein condensates in a double well potential. For large separations between the wells the position of the corresponding interference fringes are sensitive to the potential [24].

In the last part of the Letter we discuss the large $z$ behavior of the out of equilibrium force. In this limit only values $q \approx 1$ are important in the evaluation of the integral (8). By making the substitution $q^{2}-1=t^{2}$, and the $t \ll 1$ expansion, we find that the force (8) exhibits the nontrivial asymptotic behavior

$$
F_{\mathrm{th}}^{\mathrm{neq}}(T, 0, z)_{z \rightarrow \infty}=-\frac{\hbar \alpha_{0}}{z^{3} \pi c} \int_{0}^{\infty} d \omega \frac{\omega}{e^{\hbar \omega / k_{B} T}-1} f(\omega) .
$$

Notice that the force exhibits a slower $1 / z^{3}$ decay with respect to the one holding at thermal equilibrium where it decays like $1 / z^{4}$ [see Eq. (5)]. In the above equation we have introduced the function

$$
f(\omega)=\left\{|\varepsilon(\omega)-1|+\left[\varepsilon^{\prime}(\omega)-1\right]\right\}^{1 / 2} \frac{2+|\varepsilon(\omega)-1|}{\sqrt{2}|\varepsilon(\omega)-1|},
$$

which depends on the optical properties of the substrate. For temperatures much smaller than the energy $\hbar \omega_{c} / k_{B}$, where $\omega_{c}$ is the lowest characteristic frequency of the dielectric substrate, we can replace $f(\omega)$ with its low frequency limit $\left(\varepsilon_{0}+1\right) / \sqrt{\varepsilon_{0}-1}$. The force (9) felt by

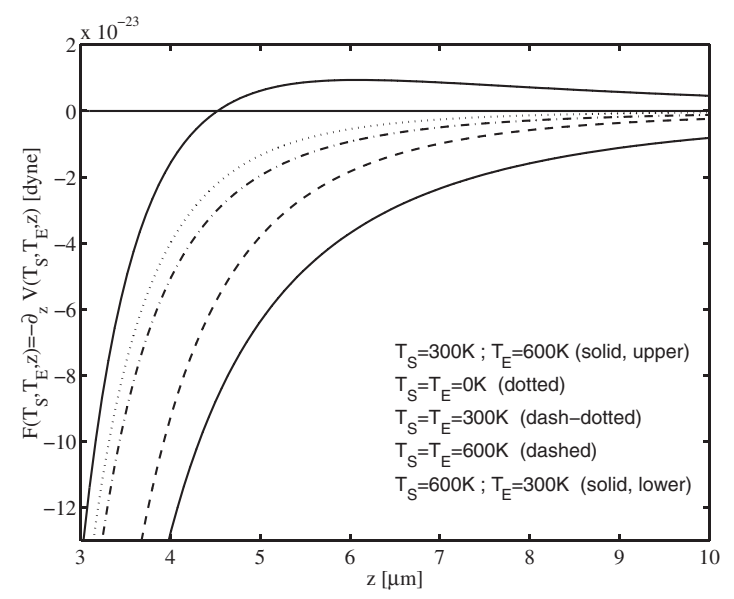

FIG. 2. Surface-atom force $F^{\text {neq }}(z)$ calculated from Eq. (9), for different thermal configurations. 


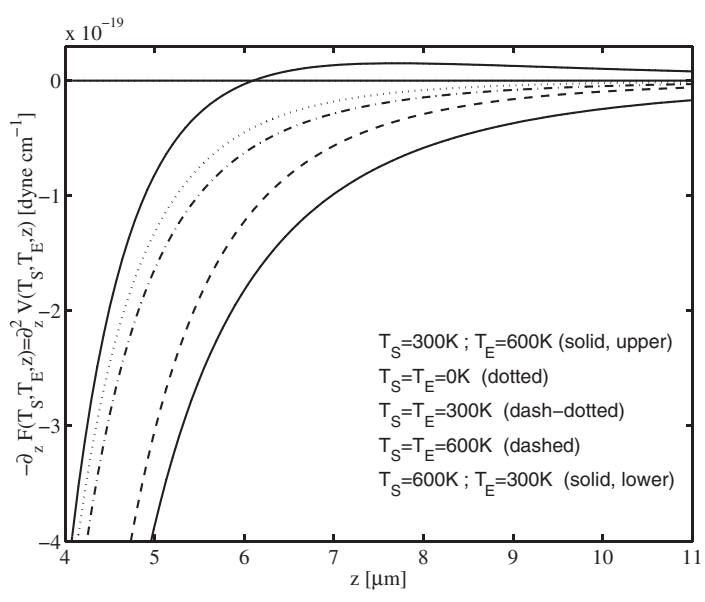

FIG. 3. Surface-atom gradient of the force $\partial F^{\text {neq }}(z) / \partial z$ calculated from Eq. (9), for different thermal configurations.

the atom then approaches the asymptotic behavior

$$
F^{\mathrm{neq}}\left(T_{S}, T_{E}, z\right)_{z \rightarrow \infty}=-\frac{\pi}{6} \frac{\alpha_{0} k_{B}^{2}\left(T_{S}^{2}-T_{E}^{2}\right)}{z^{3} c \hbar} \frac{\varepsilon_{0}+1}{\sqrt{\varepsilon_{0}-1}},
$$

holding at low temperature and at distances larger than $\lambda_{T} / \sqrt{\varepsilon_{0}-1}$ where $\lambda_{T}$ is the thermal photon wavelength calculated at the relevant temperatures $T_{S}$ and $T_{E}$ [25]. Equation (12) shows that, at large distances, the new force is attractive or repulsive depending on whether the substrate temperature is higher or smaller than the environment one. Furthermore, it exhibits a stronger temperature dependence with respect to equilibrium and contains explicitly the Planck constant. The new dependence of $F^{\text {neq }}(T, 0, z)$ on temperature and distance can be physically understood by noticing that the main contribution to the $z$ th dependent part of the electric energy $U_{E}$ arises from the blackbody radiation impinging on the surface in a small interval of angles, of order of $\left(\lambda_{T} / z\right)^{2}$, near the angle of total reflection. This radiation creates slowly damping evanescent waves in vacuum. As a result $F^{\text {neq }}(T, 0, z)$ turns out to be, in accordance with Eq. (12), of order of $-\left(\lambda_{T}^{2} / z^{3}\right) U_{B B}$, where $U_{B B} \propto T^{4}$ is the energy density of the blackbody radiation.

Equation (12) holds for a dielectric substrate where $\varepsilon_{0}$ is finite. For a metal, if one uses the Drude model, one has $\varepsilon^{\prime \prime}(\omega)=4 \pi \sigma / \omega$ with the real part $\varepsilon^{\prime}(\omega)$ remaining finite as $\omega \rightarrow 0$ so that one finds $f(\omega) \rightarrow \sqrt{\varepsilon^{\prime \prime}(\omega) / 2}=$ $\sqrt{2 \pi \sigma / \omega}$. At low temperatures Eq. (10) then gives rise to a different temperature dependence

$$
F^{\mathrm{neq}}\left(T_{S}, T_{E}, z\right)_{z \rightarrow \infty}=-\frac{\alpha_{0} \zeta(3 / 2) \sqrt{\sigma} k_{B}^{3 / 2}\left(T_{S}^{3 / 2}-T_{E}^{3 / 2}\right)}{z^{3} c \sqrt{2 \hbar}},
$$

where $\zeta(3 / 2) \sim 2.61$ is the usual Riemann function.
In conclusion, in this Letter we have calculated the surface-atom force out of thermal equilibrium and pointed out the occurrence of a new asymptotic behavior at large distances. Our predictions could be tested in experiments with ultracold atomic gases trapped close to the surface of a substrate.

We are grateful to E. Cornell, J. Obrecht, J. McGuirk, and D. M. Harber for many useful comments. It is also a pleasure to thank I. Carusotto, C. Henkel, and S. Reynaud for insightful discussions. Financial support from MURST is acknowledged.

[1] J.F. Babb et al., Phys. Rev. A 70, 042901 (2004).

[2] M. Antezza, L. P. Pitaevskii, and S. Stringari, Phys. Rev. A 70, 053619 (2004).

[3] H. B. Chan et al., Phys. Rev. Lett. 87, 211801 (2001).

[4] S. Dimopoulos and A. A. Geraci, Phys. Rev. D 68, 124021 (2003); R.S. Decca et al., Ann. Phys. (N.Y.) 318, 37 (2005).

[5] C. I. Sukenik et al., Phys. Rev. Lett. 70, 560 (1993).

[6] A. Landragin et al., Phys. Rev. Lett. 77, 1464 (1996).

[7] F. Shimizu, Phys. Rev. Lett. 86, 987 (2001); H. Oberst et al., Phys. Rev. A 71, 052901 (2005).

[8] Y. J. Lin et al., Phys. Rev. Lett. 92, 050404 (2004).

[9] T. A. Pasquini et al., Phys. Rev. Lett. 93, 223201 (2004).

[10] D. M. Harber, M. Obrecht, J. M. McGuirk, and E. A. Cornell, cond-mat/0506208 [Phys. Rev. A (to be published)].

[11] I. Carusotto et al., Phys. Rev. Lett. (to be published).

[12] H. B. G. Casimir and D. Polder, Phys. Rev. 73, 360 (1948).

[13] I. E. Dzyaloshinskii, E. M. Lifshitz, and L. P. Pitaevskii, Adv. Phys. 38, 165 (1961).

[14] P. Milonni, The Quantum Vacuum: An Introduction to Quantum Electrodynamics (Academic Press, Boston, 1994); K. A. Milton, J. Phys. A 37, R209 (2004).

[15] E. M. Lifshitz, Dokl. Akad. Nauk SSSR 100, 879 (1955).

[16] S. M. Rytov, Y. A. Kravtsov, and V. I. Tatarskii, Elements of Random Fields, Principles of Statistical Radiophysics (Springer, Berlin, 1989), enlarged edition of Rytov's original book published in Russian in 1953.

[17] C. Henkel, K. Joulain, J.-P. Mulet, and J.-J. Greffet, J. Opt. A Pure Appl. Opt. 4, S109 (2002).

[18] A. E. Cohen and S. Mukamel, Phys. Rev. Lett. 91, 233202 (2003).

[19] J. P. Gordon and A. Ashkin, Phys. Rev. A 21, 1606 (1980).

[20] J. M. Wylie and J. E. Sipe, Phys. Rev. A 30, 1185 (1984); J. E. Sipe, J. Opt. Soc. Am. B 4, 481 (1987).

[21] C. Henkel et al., Opt. Commun. 186, 57 (2000).

[22] M. Antezza (to be published).

[23] G. Roati et al., Phys. Rev. Lett. 92, 230402 (2004).

[24] Y. Shin et al., Phys. Rev. Lett. 92, 050405 (2004).

[25] The static limit, which is obtained by replacing $\varepsilon(\omega)$ with $\varepsilon_{0}$, can also be used to simplify the evaluation of Eq. (8). In the case of a sapphire substrate at ordinary temperatures $\left(\varepsilon_{0}=9.4\right)$ this approximation is very accurate at all distances. 